\title{
Un reto cultural de la actualidad: la preservación de la cuentería popular latinoamericana
}

\author{
MARIANELA Rosa PEÑA LORA*
}

\begin{abstract}
Artículo recibido: 28 de febrero de 2014
Artículo aprobado: 28 de abril de 2014

Doi: dx.doi.org/10.12804/desafios26.02.2014.08

Para citar este artículo: Peña Lora, M. R. (2014). Un reto cultural de la actualidad: la preservación de la cuentería popular latinoamericana. Desafíos, 26 (2), 217-236. doi: dx.doi. org/10.12804/desafios26.02.2014.08
\end{abstract}

\section{Resumen}

La autora aborda la relación existente entre las tradiciones y expresiones vivas en su manifestación de ámbito del patrimonio inmaterial, y la cuentería popular como una concreción, adentrándose en el análisis de los argumentos de pertenencia de esta última a la literatura y tradición oral, determinando las características generales y diferencias de ambas categorías, al tiempo que establece los fundamentos de la génesis y procedencia de la cuentería popular respecto a ellas. El análisis de la situación de empobrecimiento y declinación de la literatura oral en América Latina, ante el proceso de difusión de formas y modelos culturales provenientes de los centros nacionales y extranjeros del poder económico, como causa fundamental, a lo que no queda exenta la cuentería popular, y partiendo de la importancia de esta en el proceso de identidad cultural, demuestra la necesidad de su preservación como un gran reto cultural en la época actual. Ello permite la afirmación de que los vínculos que se establecen entre estos procesos son culturales y sociales. Culturales, por la naturaleza intrínseca y per-

\footnotetext{
* Investigadora-Promotora Cultural. Casa del Caribe, Santiago de Cuba, Cuba. Correo electrónico: peamarianela@gmail.com
} 
tenencia de ambos procesos a la esfera de la cultura, y sociales, por cuanto se establece una relación directa entre uno y otra desde las estructuras socioeconómicas existente.

Palabras clave: Patrimonio cultural inmaterial, cuentería popular, literatura oral, tradición oral, cuentero popular.

\title{
A Cultural Challenge Today: The Preservation of American Popular Storytelling
}

\begin{abstract}
The author discusses the existing relationship between the traditions and the expressions in the area of intangible cultural heritage, and popular storytelling as one of its forms. In particular, the article analyzes the arguments in terms of the affiliation of popular storytelling to literature and oral traditions, determining the general characteristics and differences of the two genres. In doing so, it establishes the fundaments of the genesis and background of popular storytelling with regard to those genres. The analysis of the situation of impoverishment and decline of oral literature in Latin America, in view of the process of diffusion of cultural forms and models stemming from national and international centers of economic power as the fundamental cause, has also affected popular storytelling. In addition, the article - based on the importance of this situation for the process of cultural identity - shows the need for the preservation of popular storytelling as a major cultural challenge in the current age. This leads to the claim that the links between these processes are cultural and social. Cultural because of the intrinsic nature and belonging of both processes to the sphere of culture; social because of the fact that there is a direct relationship between one and the other in terms of the existing socio-economic structures.
\end{abstract}

Keywords: Intangible cultural heritage, popular storytelling, oral literature, oral tradition, popular storyteller.

\section{Um reto cultural da atualidade: a preservação da contação popular de histórias latino-americana}

\section{Resumo}

A autora aborda a relação existente entre as tradições e expressões vivas em sua manifestação de âmbito do patrimônio imaterial, e a contação popular de histórias como 
uma concreção das mesmas, se adentrando na análise dos argumentos de pertença desta última à literatura e tradição oral, determinando as características gerais e diferenças de ambas as categorias, ao mesmo tempo em que se estabelecem os fundamentos da gênese e procedência da contação popular de histórias com respeito a elas. A análise da situação de empobrecimento e declinação da literatura oral n América Latina, ante o processo de difusão de formas e modelos culturais provenientes dos centros nacionais e estrangeiros do poder econômico, como causa fundamental, ao que não fica isenta a contação popular de histórias, e partindo da importância desta no processo de identidade cultural, demonstra-nós a necessidade de sua preservação como um grande reto cultural na época atual. Isso permite a afirmação de que os vínculos que se estabelecem entre estes processos são culturais e sociais. Culturais por natureza intrinseca e pertença de ambos os processos à esfera da cultura, e sociais por quanto se estabelece uma relação direta entre uma e outra desde as estruturas sócio-econômicas existente.

Palavras chave: Patrimônio cultural imaterial, contação popular de histórias, literatura oral, tradição oral, contador popular de histórias.

El enfoque central de este artículo se encuentra en tres preguntas simples: ¿qué se entiende por patrimonio cultural inmaterial? ¿Cómo definir el concepto de cuentería popular? Y ¿qué aportan estos entendimientos para pensar en la preservación de la cuentería popular latinoamericana?

\section{El patrimonio cultural inmaterial. El reto de su preservación}

En el mundo globalizado de hoy, asistimos a una creciente patrimonialización de nuevos objetos, prácticas, dominios y expresiones culturales que se fundamenta en la lógica de buscar nuevos recursos que sean susceptibles de generar riqueza y mantener el desarrollo sostenible. Como señala Gómez (2005), el interés por el patrimonio empieza a cobrar fuerza a partir de la aparición de los primeros síntomas de la crisis ecológica en el mundo, y ante la limitación existente de los recursos naturales.

Con ello, se ha ido ampliando progresivamente el estudio del patrimonio cultural, objeto del cruce de distintas disciplinas y conceptos de 
índole antropológica, sociológica, histórica, ética, etc. en la demanda de la conservación de ámbitos que transitan desde los del patrimonio histórico, arqueológico o artístico hasta la conceptualización de nuevas categorías patrimoniales de más reciente creación, como sucede con el patrimonio industrial o la nueva conceptualización del patrimonio cultural inmaterial (Gili, 2005; Gómez, 2005; Hernández, 2006; Badenes, 2006; Asiáin, 2007; Sanz, 2011).

Las convenciones de la Organización de las Naciones Unidas para la Educación, la Ciencia y la Cultura (Unesco) acerca de la cultura se han decretado como una revelación de las solicitudes de la comunidad internacional, en el establecimiento de normas internacionales dispuestas a servir de base a la formulación de políticas culturales y el fortalecimiento de la colaboración. Los ocho instrumentos normativos obtenidos en los 55 años de largo bregar muestran las prioridades culturales, y al confrontarlas se puede distinguir la marcha de estas políticas y el rol desplegado por los diversos actores influyentes.

Estas normativas se integran entre sí, en una dialéctica que parte de la diversidad de problemáticas, al mismo tiempo que permiten un proceso de perfeccionamiento continuo, ya que manifiestan el impacto de las políticas anteriores en relación con las nuevas urgencias. En su conjunto, estas herramientas proporcionan los medios precisos para ayudar a los Estados miembro a resguardar la pluralidad cultural del orbe en un contexto internacional en constante desarrollo y su validez se sustenta en el deber de aplicarlos cuando se legalizan.

Así la historia, el patrimonio cultural, en sus inicios, estuvo relacionado con la magnificencia material, concepción que fue ampliada desde la exclusiva materialidad hacia la integración de los valores "inmateriales" de la cultura, que hasta ese momento no tenían relevancia.

La Conferencia General de la Unesco aprobó la Convención para la Salvaguardia del Patrimonio Cultural Inmaterial en su 32 a reunión, efectuada en 2003. La aprobación de la Convención marcó un hito en la perfeccionamiento de las políticas internacionales de impulso de la diversidad cultural, ya que por primera vez la comunidad internacional 
distinguía la necesidad de prestar apoyo a un tipo de manifestaciones y expresiones culturales que hasta entonces había carecido de un marco jurídico y programático de esa trascendencia.

Adicionada a otros instrumentos internacionales concernientes al patrimonio cultural, la Convención de 2003 tiene por objeto primordial salvaguardar los usos, las representaciones, las expresiones, los conocimientos y las técnicas que las comunidades, los grupos y, en algunos casos, los individuos reconocen como parte integrante de su patrimonio cultural. Este patrimonio se manifiesta en los siguientes ámbitos: a) tradiciones y expresiones orales, incluido el idioma como vehículo del patrimonio cultural inmaterial; b) artes del espectáculo; c) usos sociales, rituales y actos festivos; d) conocimientos y usos relacionados con la naturaleza y el universo, y e) técnicas artesanales.

Pese a su fragilidad, estos ámbitos constituyen un importante factor del mantenimiento de la diversidad cultural frente a la progresiva globalización, que contribuye al diálogo entre culturas y promueve el respeto hacia otros modos de vida; y su importancia no reside en la expresión cultural en sí, sino en el cúmulo de conocimientos y técnicas que acerca de este se transmiten de generación en generación. El valor social y económico de esta transmisión de conocimientos es acertado para los grupos sociales tanto minoritarios como mayoritarios de un Estado, y reviste la misma importancia para los países en desarrollo que para los países desarrollados: "Esa misma mirada vale para todo aquello que no tiene una forma material y que, sin embargo, nos arropa en un patrimonio cultural de las identidades, los sentimientos compartidos y los futuros imaginados" (Arizpe, 2009, p. 18).

La concepción del carácter integral del patrimonio inmaterial, viéndolo como un resultado - consecuencia del diálogo intercultural y de la relación de la comunidad con el medio - y analizando adecuadamente sus raíces, sus perspectivas, sus nexos e interdependencias en la metamorfosis del mundo de hoy, reviste importancia raigal, por cuanto vivimos tiempos en los que la identidad de los pueblos se ve amenazada ante el proceso desmedido de globalización mundial. De ahí el reto de su preservación sustentada en su carácter: 
- Tradicional, contemporáneo y viviente a un mismo tiempo, por cuanto no solo incluye tradiciones heredadas del pasado, sino usos rurales y urbanos contemporáneos propios de diversos grupos culturales.

- Cohesionador: el patrimonio cultural inmaterial no se presta a preguntas sobre la pertenencia de un determinado uso a una cultura, sino que contribuye a su cohesión social, promoviendo un sentimiento de identidad y compromiso que permite que los individuos se sientan miembros de una o varias comunidades y de la sociedad en general.

- Representativo: ya que no se valora simplemente como un bien cultural, a título comparativo, por su exclusividad o valor excepcional, sino que florece en las comunidades y depende del proceso de trasmisión al resto de la comunidad, de generación en generación, o a otras comunidades.

- Basado en la comunidad: solo se considera un patrimonio si es reconocido como tal por las comunidades, grupos o individuos que lo crean, mantienen y transmiten. Sin este reconocimiento, nadie puede decidir por ellos mismos que una expresión o un uso determinado forman parte de su patrimonio.

La inclusión de las tradiciones o expresiones vivas heredada de nuestros antepasados y transmitidas a nuestros descendientes en el concepto de patrimonio inmaterial nos conduce al término que abarca y define esas tradiciones y expresiones vivas en su versión literaria, siendo este el de literatura oral y tradiciones orales, por cuanto constituyen su puente y reservorio (a su vez de la cuentería popular), núcleo de nuestro estudio. Revelar esta relación se constituye aquí en un elemento fundamental.

\section{Tradición y literatura oral: conceptos de un mismo orden}

Comencemos por el primer término: literatura oral, el cual fue creado por Paul Sébillot en un proceso de recopilación de relatos, mitos, le- 
yendas, cuentos, proverbios, cantos y otros géneros del acervo oral tradicional de la Alta Bretaña, y publicado en un libro en 1881.

Es un término polémico que ha llamado a discusión teórica a muchos investigadores y representantes de diversas ciencias: los que no comparten su viabilidad (Ong, 1982) y otros o en su defecto, utilizan el término oralitura (Matos, 1990; Daroqui, 1993; Ancalao, 2010; Bansart, 1989; Melgar, 2007), por citar algunos, se sustentan en lo general, en referentes etimológicos, vinculados a la palabra literatura y su procedencia del latín littera, que significa la letra del alfabeto, lo que representa para ellos una contradicción flagrante. Matos al respecto señala:

Ellos no son "literatura", son una forma expresiva que puede ser incluida simultáneamente en dos agregados mayores (de existencia meramente analítica): las artes verbales y las artes escénicas. En tanto artes verbales, muchos de estos desempeños exhiben atributos estéticos susceptibles de una apreciación y valoración literarias, pero eso no puede llevar a clasificarlos como fenómenos "literarios"; hacerlo es cometer un atropello cultural, es un reduccionismo, porque al hacerlo se suprimen los restantes rasgos expresivos (1989, p. 44).

Colombres (1997), en oposición a los renuentes al término y en específico refiriéndose a Ong (1982), declara que a este le pareció monstruoso hablar de una literatura oral, al considerar que sería referirse a una cosa en términos de otra o presentar la oralidad como una variante de la escritura, y aun considerando que desde un purismo conceptual podría ser cierto, el autor alega con un justo razonamiento - con lo cual coincide la autora - que las palabras no son solamente su etimología, ya que, más que ello, lo importante son los significados que históricamente se les asignan.

Con respecto a los que proponen el término de oralitura como sustituto de literatura oral, señala también Colombres, que esta expresión nos conduciría a guardar dicha producción en una gaveta que difícilmente será incluida en lo artístico, por cuanto la escritura no accedería a sentarse a la mesa de ese "banquete". Agrega que las bellas letras definen 
con un sentido excluyente, de dominación, la producción narrativa y lírica de las culturas orales, la que resulta así segregada y puesta al lado de los subproductos literarios del sistema dominante. De ahí que reclame que si se quiere reivindicar la seriedad de la producción narrativa y lírica de la oralidad, no se puede renunciar a ese bastión con reputación que resultó el concepto de literatura tan solo por un hábito etimológico (Colombres, 1997, p. 15).

Esta noción ha tenido en Stith Thompson a uno de sus mayores teóricos y difusores. En su documentado y concienzudo estudio, consideró el cuento folclórico o cuento oral como una de las "formas de la literatura oral” (1972, p. 22). También Monsonyi (1990, p. 6), en ese mismo sentido, considera que "literatura" es cualquier conjunto de textos de suficiente relevancia sociocultural que forme parte del patrimonio cognitivo y afectivo de un grupo humano determinado y que sea transmitido de generación en generación de forma ininterrumpida, aunque sujeta a cambios diacrónicos: "En este contexto parece secundario si, tal material es oral o escrito".

Con respecto a la tradición oral, esta se ha abordado desde diferentes perspectivas: una de ellas es el enfoque literario (de interés de la autora), que asume la tradición como una composición creativa del narrador, tal como lo exponen Gloria Vergara (2004) y Eugenia Revueltas y Herón Pérez Martínez (1992). Otras vertientes se asoman a este término desde el punto de vista histórico: Sebe (2008) y otros enfoques son develados desde la mirada de construcción sociocultural (Figueroa, 2008).

Jan Vansina (1997), autor clásico en este tema, realizó grandes aportes y la definió como: "todos los testimonios orales, narrados, concernientes al pasado [...] no todas las fuentes orales son tradiciones orales. Solo lo son las fuentes narradas; es decir, las que son transmitidas de boca en boca por medio del lenguaje" (1997, pp. 33 y 34).

Hampaté (1982, pp. 186 y 187), un estudioso africano del tema, considera que la tradición oral no se limita a cuentos y leyendas o a relatos míticos e históricos. Para él, es la gran escuela de la vida. Es religión, 
historia, recreación y diversión. En América, y en otros lugares del mundo como en África, muchas de sus sabidurías han permanecido en la memoria y se han expresado en mitos, cuentos y cantos o en narraciones épicas. También Edgardo Civallero sintetiza magistralmente su concepto y función social cuando expresa:

La tradición oral es un fenómeno rico y complejo, que se convirtió en el medio más utilizado — a lo largo de los siglos_ para transferir saberes y experiencias. Sus múltiples definiciones coinciden en señalar que representa la suma del saber [...] que una sociedad juzga esencial y que, por ende, retiene y reproduce a fin de facilitar la memorización, a través de ella la difusión a las generaciones presentes y futuras (2007, p. 67).

Otros autores como Fribourg (1987) establecen la diferencia entre literatura oral y tradición oral, considerando que son términos que no se pueden confundir y señalando que la literatura oral no es más que una parte de la tradición oral. Según Genevieve Clame-Griau, citada por Fribourg, la literatura oral es "la puesta en forma, regulada por un código propio de cada lengua y de cada sociedad, de un fondo cultural" (1987, p. 1).

La constatación de estos aspectos nos indica, en primer lugar, que la tradición oral se redefine por su propia dinámica interna, determinada por la apropiación de elementos socioculturales que entran a formar parte de su enriquecimiento constante. En segundo lugar, nos remite al conocimiento de que la literatura oral es elemento constitutivo de la tradición oral, aquel segmento que toma forma literaria, con una estructura propia del grupo que la produce, y en función de ello obedece a ciertas reglas de expresión propias de este grupo, lo que hace afirmar que un hecho de relato oral que no se adapta al patrón y que no obedece a ciertas condiciones de expresión podría desprenderse de la tradición oral, no de la literatura oral (Fribourg, 1987).

En consecuencia, lo importante de este análisis es la reafirmación de que la literatura y la tradición oral, aunque no coincidentes son conceptos de un mismo orden, subsumido uno en el otro - literatura oral 
en tradición oral- e implicados ambos en los procesos narrativos históricamente y en la actualidad.

En correspondencia con los objetivos de nuestra investigación, de gran alcance resultan los criterios acerca de la estructura de la literatura oral y, dentro de ello, autores como Vansina (1997), quien elaboró una de las tipologías más completas y útiles, manifestadas en cinco grupos principales, dentro de la cual destacan los relatos contentivos de cuentos, mitos y leyendas; así como Monsonny (1990), Asiáin (2007) y Trapero (2005), quienes desde sus posicionamientos han señalado también el género narrativo en prosa como reservorio de los cuentos, las leyendas o las narraciones míticas y otros.

De forma general, la narrativa oral es un género discursivo universal por medio del cual las personas intentan darle sentido a su experiencia, en correspondencia con los determinados contextos de actuación que concretan la forma en que se realiza y sus variaciones culturales ( $\mathrm{Za}$ bala, 2006). Esto quiere decir que las nociones de verdad, estilo y lenguaje que son consideradas apropiadas; la forma en que se conectan las ideas y se secuencian los eventos; las partes en que esta está compuesta, y los patrones de interacción que la producen (el rol del que la cuenta y el de la audiencia, por ejemplo), son diversos en correspondencia con los distintos contextos culturales.

\section{Breve referencia a la literatura oral en el contexto latinoamericano y del Caribe en la actualidad}

Dentro del contexto latinoamericano y del Caribe, la literatura oral tiene una riqueza ampliamente reconocida, dada la historia de encuentros culturales (indoamericanas, afroamericanas, hispanoamericanas y mestizas) que existieron y coexisten actualmente. Por eso su expresión constituye síntesis de la cosmogonía africana, europea y precolombina, generadora de un caudal cultural que se hace materia de intenso análisis (Montoya, 2004). Acerca de este proceso se refiere Lienhard expresando:

Hasta la generalización reciente de los medios de comunicación audiovisuales, la realidad mayoritaria del ejercicio de la literatura 
en el subcontinente ha sido, sin la menor duda, la práctica oral —de las subsociedades indígenas, mestizas o negroides, del campesinado pobre, de los sectores urbanos marginales. Esta práctica, en rigor, no puede conocerse sin ser vivida in situ, es decir, en las mil y una comunidades donde se desarrolla: tarea a todas luces vedada a un investigador, aún a un grupo de investigadores, salvo en casos contados. La literatura escrita híbrida, en cambio, más accesible a la investigación científica, ofrece siquiera a unos atisbos de lo que pudo ser y es todavía el continente sumergido de las literaturas orales; al mismo tiempo, su propia existencia atestigua que entre los dos universos, el de la escritura y el de la oralidad, siempre ha habido zonas de contacto, de conflicto, de intercambio (1990, p. 58).

Ello constituye una de sus más importantes regularidades: la diversidad de componentes etnoculturales que de manera impuesta o voluntaria migraron hacia el llamado Nuevo Mundo, mestizándose en un complejo proceso cuyas singularidades están selladas, entre otras variables, por las tipicidades regionales que hoy en gran medida se mantienen, por lo que no es posible hablar de una literatura oral latinoamericana homogénea, sino de literaturas latinoamericanas, distintas según subregiones y países, y dentro de ellos, según clases sociales y culturas étnicas, en correspondencia con sus propios procesos civilizatorios. De tal manera se han afianzado estas diferencias que, por citar un ejemplo, la oralidad sigue siendo el soporte de toda creación literaria para más de veinticinco millones de indígenas en Latinoamérica repartidos por todo el continente (Petrich, 2009), lo que no escapa a la aplicación violenta y discriminatoria de un modelo supuestamente universal (europeo) que desprecia la tradición oral de las literaturas indígenas (Pulido, 2009).

Tal y como expresa Petrich (2009), hoy no solo tenemos la coyuntura de oír mitos y cuentos atesorados desde el periodo prehispánico, sino que concurrimos a la creación de otros géneros y a la inscripción de nuevos temas. En realidad, a pesar de un acceso más o menos afirmado a la escritura, a través de la oralidad se crean y transmiten los discursos artísticos, y allí es donde se debe indagar acerca de la originalidad creativa de estos grupos, su permanencia y su actualidad. 
Es sabido que en las sociedades que conocen la escritura, la tradición oral pasa a ser vía de expresión de las culturas subalternas o, lo que es lo mismo, las prácticas significantes propias de las oralidad verbal adquieren capacidad para la resistencia cultural, dada su condición de reducto, reservorio y trinchera, situación entronizada en toda Hispanoamérica.

Se conoce que el cuadro socioeconómico que presenta América Latina y el Caribe en la actualidad es complejo y diverso, de manera que muchos autores afirman que nos encontramos en una encrucijada cultural en ese orden (Lara, 1993). La difusión de formas y modelos culturales provenientes de los centros nacionales y extranjeros del poder económico ha provocado, efectivamente, un empobrecimiento de la actividad creativa de las grandes mayorías nacionales, que incluye tanto a los grupos y etnias que aún retienen prácticas culturales de tradición ancestral de la ciudad y del campo (muchos de ellos en el campo han emigrado hacia las ciudades, alejados de sus comunidades e integrados a su hábitat a través de una cultura de sobrevivencia) como a las burguesías urbanas, las cuales, a pesar de contar con una cultura o por lo menos una educación que se considera más sólida que la etnias y población rural en general, llegan a ser mucho más propicias a la desnacionalización (Mariscal, 1990).

En muchos casos, esa parte de la población que en sus maneras de expresión artística aún retienen ciertas rasgos distintivos y peculiaridades de realización artesanal, en las que al no establecerse diferencias radicales entre productor y consumidor, los patrones heredados del pasado son descifrados y regenerados en términos propios y no en términos de un consumidor distante al ámbito cultural en el que se originan (Mariscal, 1990).

Dentro de esta generación artística "artesanal" se encuentran los diversos géneros y formas literarias que se transmiten oralmente. Los mitos, los cuentos, las leyendas, etc. de origen no "letrado" que se comunican de generación en generación por vía oral, que si bien van perdiendo cada vez más pujanza frente a formas seudoliterarias destinadas al consumo popular, procedente tanto del extranjero como 
de los centros nacionales del poder económico, no han desaparecido totalmente.

Esta narrativa oral de carácter tradicional — constituida por un proceso de reelaboración y recreación de los marcos y contextos actuales- ha dado lugar a la llamada cuentería popular, categoría que actualiza en el hoy y en Latinoamérica el término literatura oral, en cuanto al género de narrativa se refiere, y desde lo teórico es una lectura y mirada prototípica en ese orden, como expresión orgánica de la identidad, que contribuye de manera positiva a la cohesión, a la evolución dinámica y a la durabilidad de esa cultura que representan.

Esta cuentería se caracteriza por ser herencia e innovación, ya que responde tanto a la pretensión de transmitir el saber histórico de la comunidad como al de ofrecer comentarios y soluciones a problemas de actualidad. Da cuenta de una conciencia colectiva concerniente a un sujeto transindividual (Lara, 1993) o sujeto colectivo (Ballón, 1995), que conjuntamente con la individualidad- que depende del ingenio y estado de ánimo del narrador, y con el contexto en el que este se desarrolla-, permite que las tradiciones se generen constantemente a medida que se van adaptando al proceso de expresión de esa conciencia colectiva y que las palabras del pasado lleguen a coincidir con los valores e imágenes del presente asumiendo nuevos significados.

Es una literatura popular, generalmente de carácter anónimo y tradicional, es decir, que el pueblo la hace suya en el olvido de su autor, y la somete a reelaboraciones y modificaciones. En el relato de cada cuentero aparecen las visiones compartidas por su grupo, aquellas tradiciones y lecturas de la realidad que se acumulan y sedimentan en torno a narrativas nuevas y viejas, formas propias de verse y narrar la propia comunidad.

En esas condiciones, esta cuentería está representada por los cuenteros populares que la literatura recoge en ocasiones como narradores folclóricos o tradicionales, artistas orales tradicionales (Monsonyi, 1990) de filiación fundamentalmente campesina, que incluye a mineros, 
pescadores y representantes de las capas pobres de la población rural, urbana o semiurbana.

La Unesco, a través la inclusión del patrimonio inmaterial y de la designación de la categoría de tesoros humanos vivos, acude a ellos como los portadores-intérpretes de la tradición oral, y de esa manera acentúa los rasgos corporales de la oralidad en general hasta entenderla como expresión corporal y personal de esos portadores-intérpretes.

En esta referencia al cuentero popular, es significativa la aclaración de que con este término no se alude a los narradores orales que fomentan y practican la narración oral escénica, que como concepto se extiende por el mundo colmando los escenarios y se impone como un arte escénico más (Garzón, 1995). Se trata de ese hombre humilde que - llámese chamán ("curandero de la palabra", en casi todas las culturas originarias); aedo; juglar, bardo o trovador; griot en África; fabulador en Arabia; tusitala en Polinesia; penglipurlara en Malasia; seanchaí en Irlanda; trovero del sertón brasileño; cacheros del oriente venezolano, en fin, cuentero en toda América- demuestra que el origen del arte de contar cuentos es ancestral y hace que la cuentería popular se convierta en un diapasón penetrante en las esferas de la identidad, cultura y resistencia.

Se trata de esa legión de hacedores de cultura que, mediante un proceso de resimbolización de rituales y del capital cultural heredado y acumulado por la memoria histórica, tiene la misión de conservar, por medio de cuentos y narraciones cotidianas, el lenguaje y al mismo tiempo de proponer nuevas combinatorias simbólicas como formas de resolver conflictos de orden social y cultural en general. Ese hombre que en estos tiempos se constituye, al decir de Néstor García Canclini (1990), en una manifestación de la hibridez como nueva manera de pensar los mestizajes culturales.

Nos referimos a esa institución auténtica y legendaria que permite que lo oral se constituya como punto de encuentro de historias para compartir las experiencias. A esa figura de la cuentería popular que hace que las etnias, pueblos y nacionalidades se reconozcan primero como 
colectividad, lo cual permite que su cultura sea dinámica y creativa y que a partir de este intercambio de relatos orales, el proceso social que se desarrolla sea una experiencia donde se crean y valoren todos los elementos que forman parte de ella sin exclusión ni marginación (Ramírez, 2005).

Su existencia y reconocimiento en el presente latinoamericano nos conduce al razonamiento de que estamos llegando a un punto de nuestro devenir colectivo en el cual la diversidad de expresiones orales estéticamente marcadas converge, desde todos los tiempos y lugares, para convertirse en un patrimonio común de la humanidad; de ahí que sea inaplazable el rescate de este sujeto y proceso, y con ello estimular el desenvolvimiento de la oralidad tanto tradicional como de carácter más innovador.

Algunos investigadores se han dedicado al estudio de los cuenteros populares desde una perspectiva antropológica y etnográfica. Citamos a Daniel Mato (1989), en Venezuela, quien mediante observaciones de campo examinó los casos de casi setenta narradores venezolanos de más de treinta comunidades de diversas tradiciones culturales, y mediante investigación documental, otras diez comunidades de Venezuela (Mato, 1989). Adicionalmente, comprobó su importancia en más de treinta grupos sociales, correspondientes a diversas demarcaciones culturales y sociales en los cinco continentes (Mato, 1989).

Nina de Friedemann (1997), antropóloga colombiana que dedicó su vida al estudio de culturas, también penetró en los recónditos espacios colombianos en el litoral pacífico, al pie de los Montes de María, cerca de Cartagena de Indias, por los ríos del Chocó, y escribió una historia que hizo que su colaborador de tantos "soles" y su amigo de tantos años, Jaime Arocha, la despidiera con el epíteto de "Argonauta del firmamento".

En 1966, Miguel Barnet, un discípulo del pionero cubano de la "afroamericanística" —el etnólogo cubano Fernando Ortiz_, para lograr el testimonio del protagonista Esteban Montejo en su libro Biografía de un cimarrón, no escatimó la inclusión del habla del cimarrón, 
para lo cual desarrolló todo un proceso investigativo de cara a cara con sus protagonistas. María Eugenia Boito, en Argentina, con El caldero de los cuentos aboga por la cuentería popular como experiencia del sujeto y como práctica social.

Mannheim Bruce, en Perú, estudió los cuentos como una estrategia de transmisión cultural de valores en la que se desarrolla una preocupación por que la siguiente generación cuente con habilidades necesarias (cómo trabajar, cómo tener coraje y abordar el sufrimiento, etc.).

Estas son maneras sui generis de salvaguardar la cuentería popular. La que ofrece la autora es también otra forma de defensa del arte viviente de la narración oral de los de abajo y vía de expresión de las culturas subalternas.

Esas son concreciones de un vínculo de pertenencia y pertinencia del patrimonio cultural inmaterial y la cuentería popular, de carácter cultural, dado por la naturaleza intrínseca de ambos procesos dirigidos a la identidad de pueblos, etnias y naciones, y su expresión de determinación socioeconómica en los contextos de actuación y manifestación.

En sentido general, en su acepción continental, la cuentería popular expresa la noción de la americanidad como la comprensión de lo más específico del ser americano, y a partir de sus resortes esta noción, real o mítica, se constituye en criterio de búsqueda identitaria en el constante proceso de reelaboración y evolución de las visiones culturales.

La pluralidad cultural latinoamericana tiene eco en la pluralidad de los géneros de expresión, por lo que la cuentería popular, como manifestación de uno de ellos en su versión literaria, conforma una de las voces que reflejan la innovación y la continuidad espacial y temporal, al participar en el cruce de miradas sobre las distintas áreas geográficas y los periodos históricos que constituyen hitos de una construcción identitaria.

Aunque en ella es inevitable la complicidad con modelos culturales y epistemológicos occidentales, esta identidad emerge sobre la base de la 
confrontación con estos modelos y los múltiples contrastes de su otredad, que explica la típica esencia múltiple de heterogeneidad cultural, la cual no implica fragmentación sino unidad compleja, consolidada en los elementos diversos de una historia compartida y de origen común. Esta visión es la que trasciende a las diferentes y complejas visiones contrarias a la avalancha globalizadora de signo homogeneizador en esta América. Contrarrestarla es un gran reto por asumir.

\section{Referencias}

Ancalao, L. (2010). Oralitura: una opción por la memoria. Recuperado de http:// maximobombachita.blogspot.com/2010/02/oralitura.html

Arizpe, L. (2009). El patrimonio cultural inmaterial de México: ritos y festividades. México: Miguel Ángel Porrúa-Cámara de Diputados-unAM-Conaculta. Asiáin Ansorena, A. (2007). Elestudio y la preservación del patrimonio oral: bacia una antropología de la mente corporizada. Recuperado de http:/ / www.navarchivo.com/files/file/el $\% 20$ estudio $\% 20 \mathrm{y} \% 201 \mathrm{a} \% 20$ preservaci $\% \mathrm{~d} 3 \mathrm{n} \% 20$ del $\% 20$ patrimonio $\% 20$ oral.pdf

Badenes, D. (2006). Actores sociales y apropiación del patrimonio en una escala local: la universidad platense en la pugna por una memoria de lo urbano. Ponencia presentada en el VIII Congreso Internacional de Rehabilitación del Patrimonio Arquitectónico y Edificación, Centro Internacional para la Conservación del Patrimonio. Buenos Aires: CICOP. Recuperado en http://www.cicopar.com.ar/congreso/miercoles13.htm

Ballón Aguirre, E. (1995). La literatura oral en Latinoamérica: una hipótesis semiolinguística. Revista de Centros del Lenguaje (11-12), 303-355.

Bansart, A. (1989). El Caribe: identidad culturaly desarrollo. Caracas: Editorial de la Universidad de Simón Bolívar.

Boito, M. E. (2000). La importancia de la oralidad en la cultura contemporánea. Revista Latina de Comunicación Social, 35. Recuperado http://www. ull.es/publicaciones/latina/Argentina2000/21 boito.htm

Civallero, E. (2007). Notas sobre la tradición oral. Recuperado de http://tradicionoral.blogspot.com/2007/09/tradicin-oral.html

Colombres, A. (1997). Celebración del lenguaje. Buenos Aires: Del Sol.

Costa, F. (2009). Cuestionar el sentido común para investigar, investigar para cuestionar el sentido común. Entrevista a Daniel Mato. Papeles de Trabajo, 2 (5). 
Daroqui, M. (1993). Las pesadillas de la historia en la narrativa puertorriqueña. Caracas: Monte Ávila.

Figueroa Serrano, D. (2008). Tradición oral e identidad étnica en la Costa Nahua de Michoacán, México. Revista de Antropología Experimental, $2(8), 41-51$.

Fribourg, J. (1987). La literatura oral, imagen de la sociedad. Temas de Antropología Aragonesa, 1 (3), 101-111.

Friedemann, N. de (1997). De la tradición oral a la etnoliteratura: ponencia presentada en el Congreso Abra Palabra en la Universidad Tecnológica de Santander, Bucaramanga, el 4 de septiembre de 1996. Revista América Negra, 5 (13), 5-16.

García Canclini, N. (1990). Culturas híbridas: estrategias para entrary salir de la modernidad. México D. F.: Grijalbo.

Garzón Céspedes, F. (1995). El arte oral escénico de contar cuentos. Madrid: Frakson.

Gili, M. (2005). La historia oral y la memoria colectiva como herramientas para el registro del pasado. TEFROS, 2 (8), 10-19.

Gómez Pellón, E. (2005). Desarrollo sostenible, patrimonio cultural y turismo: concepciones teóricas y modelos de aplicación. En A. Santana y L. Prats (Coords.), El encuentro del turismo con el patrimonio cultural: concepciones teóricas y modelos de aplicación (pp. 71-93). Sevilla: Fundación El Monte-Federación de Asociaciones de Antropología del Estado Español-Asociación Andaluza de Antropología.

Hampaté Ba, A. (1982). La tradición viviente: historia general de África. París: Tecnos-Unesco.

Hernández Llosas, M. (2006). Diversidad cultural, patrimonio e identidad en Argentina. En La dimensión social del patrimonio (pp. 19-30). Buenos Aires: CICOP.

Lara Figueroa, C. (1985). El destino en los cuentos populares de Guatemala. Tradiciones de Guatemala (21-22), 9-31.

Lara Figueroa, C. (1993). Algunos problemas teóricos de la literatura oral. Oralidad: Anuario para el Rescate de la Tradición oral de América Latina, 5, 28-31.

Lienhard, M. (1990). La vozy su buella. La Habana: Casa de las Américas. Mannheim, B. (1999). Hacia una mitografía andina. En J. C. Godenzzi (Ed.), Tradición andina y amazónica: métodos de análisis e interpretación de textos 
(pp. 47-79). Cuzco: Centro de Estudios Regionales Andinos "Bartolomé de las Casas".

Mariscal, B. (1990). La cultura de la crisis: tradición oral urbana y fronteriza. Oralidad: Anuario para el Rescate de la Tradición Oral de América Latina, 2, 20-24.

Mato, D. (1989). Cuenteros afrovenezolanos en acción. Revista del Instituto Andino de Artes Populares, 3 (12), 43-50.

Mato, D. (1990). El arte de narrar y la noción de literatura oral: protopanorama intercultural y problemas epistemológicos. Caracas: Universidad Central de Venezuela-Consejo de Desarrollo Científico y Humanístico.

Mato, D. (1992). Narradores en acción: problemas epistemológicos, consideraciones teóricas y observaciones de campo en Venequela. Caracas: Academia Nacional de Historia-Fundación Latino.

Melgar Brizuela, L. (2007). Oralitura de El Salvador: antología de narrativa oral popular. San Salvardor: Universidad de El Salvador.

Mendizábal, R. I. (2012). La lengua y lo afro: de la literatura oral a la oralitura. Quito: Ciespal.

Monsonyi, E. (1990). La oralidad. Oralidad: Anuario para el Rescate de la Tradición Oral de América Latina, 2, 5-20.

Montoya, V. (2004). La tradición oral Latinoamericana. Letralia. Tierra de Letras, 20 (108), 9-17.

Ong, W. (1982). Oralidady escritura. México: Fondo de Cultura Económica.

Petrich, P. (2009). Actualidad de la literatura oral latinoamericana. Recuperado de http://www.lacult.org/docc/oralidad_03_31-34-actualidad-de-laliteratura.pdf

Pulido Tirado, G. (2009). El canon literario en América Latina. Signa, 18 (4), 99-114.

Ramírez, M. V. (2005). La oralidad como recurso de persistencia: el caso de las historias de las mujeres mapuches-huilliches de maihue, $\mathrm{x}$ región de los lagos. Revista de Antropología Iberoamericana, 41 (20), 78-86.

Revueltas, E. y Pérez Martínez, H. (1992). Oralidad y escritura. Zamora, México: El Colegio de Michoacán.

Sanz Abad, J. (2011). La reutilización de la narración oral como práctica cultural a partir de dos ejemplos: el maratón de cuentos de Guadalajara y la aparición de los narradores orales profesionales. Documento procedente del XII Congreso De Antropología de la Federación de Asociaciones de Antropología 
del Estado Español (FAAEE). Recuperado de http://www.antropologiacastillayleon.org/.../la\%20reutilizacion\%20de

Sebe Bom Meihy, J. C. (1992). Definiendo la historia oral. Revista Historias, 7 (29), 89-97.

Thompson, S. (1972). El cuento folklórico. Caracas: Ediciones de la Biblioteca Universidad Central de Venezuela.

Trapero, M. (2005). Identidad cultural y globalización: la literatura oral en los pueblos hispánicos. Recuperado en http://mdc.ulpgc.es/cdm/ref/collection/ asmtloc/id/6794

Vansina, J. (1997). La tradición oral: Barcelona. Barcelona: Labor.

Vergara, G. (2004). Palabra en movimiento: principios teóricos para la narrativa oral. México: Praxis-Universidad Iberoamericana.

Zabala, V. (2006). La oralidad como performance: un análisis de géneros discursivos andinos desde una perspectiva sociolingüística. Revista BIR A, 8 (33), 129-137. 